\title{
Popped eye (blunt trauma leading to penetrating eye injury and lens displacement in the pseudophakic eye)
}

\author{
P Gilligan, M J Gallagher, M Dudley, P Cryer
}

\begin{abstract}
Blunt trauma to the eye of patients with an artificial intraocular lens may cause globe rupture with all the accompanying sequelae. It must be recognised promptly and dealt with appropriately before urgent referral to ophthalmology.

(Emerg Med f 2001;18:510-511)
\end{abstract}

Keywords: ophthamology

\section{Case report}

An 84 year old woman who had undergone left intraocular lens implantation eight years earlier attended the accident and emergency department having fallen. She sustained a left Colles fracture and a laceration of the left eyebrow. On examination her left eye demonstrated a visual acuity of hand movements only. The superior cornea was penetrated with expulsion of the iris and the upper aspect of an implanted intraocular lens. There was a secondary anterior chamber haemorrhage present with an accompanying pupil irregularity (fig 1 ).

Operative notes revealed that the patient had undergone an uncomplicated left extracapsular cataract extraction with posterior chamber intraocular lens implantation eight years previously. This involved the creation of a wide corneal section superiorly to permit the expression of the cataract and to facilitate the placement of a non-foldable polymethylmethacrylate implant into the capsular bag.

Initial management of this patient included the administration of analgesia, antiemetics, intravenous antibiotics and tetanus prophylaxis. An eye shield was used for protection to prevent further accidental extrusion of the intraocular contents. The patient was subsequently transferred for ophthalmological assessment.

Reconstruction of the left anterior segment was performed. A wound swab was taken from the superior incarcerated iris for culture and

\section{Hospital, West \\ Yorkshire, UK \\ P Gilligan \\ M Dudley \\ P Cryer}

Airedale Genera

The Bradford Royal Infirmary, Bradford, UK

M J Gallagher

Correspondence to:

Dr Gilligan, 1 Far Moss,

Alwoodley, Leeds

LS17 7NU, UK

(dhegarty@ireland.com)

Accepted for publication 20 November 2000

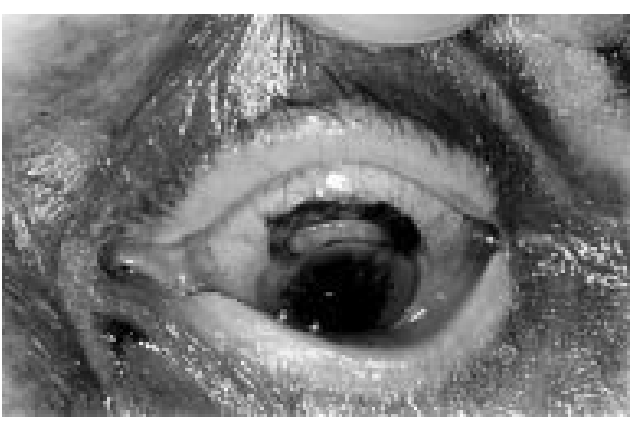

Figure 1 Protrusion of the implanted intraocular lens through the superior cornea. sensitivities. The intraocular lens was removed through the penetrated corneal section and the incarcerated iris was dissected from the wound between ten and two clock hours.

The remaining iris was retroplaced into the anterior chamber. An anterior vitrectomy was performed and $10^{\circ}$ nylon sutures used for wound closure.

Postoperatively this patient's visual acuity improved to 6/24 with pinhole and she has been subsequently listed for the placement of an anterior chamber intraocular lens implant.

\section{Discussion}

The Pub Med Database of the United States National Library of Medicine has in excess of nine million citations of Medline and PreMedline Articles. This database listed 18593 articles on eye trauma with 403 on perforating eye injuries. A search through these 403 revealed 10 relevant articles. Intraocular lens implantation, first performed 48 years ago, has revolutionised the treatment of patients with cataracts. The procedure has undergone many advances. The posterior chamber intraocular lens is now used most frequently. The iris fixated lenses are almost never used and the anterior chamber lens infrequently so. Rupture of the corneolimbic cicatrices in patients after cataract surgery is reported as happening after contusional injury. ${ }^{1}$ This most frequently happens in the first month after surgery but it has been described as happening on the day of surgery and up to 17 years after keratotomy. ${ }^{2}$

Canavan and Archer in their study on 205 patients who had suffered contusional eye injuries found that $24.5 \%$ had lens damage but most of these were not displaced. ${ }^{3}$ Margel et al in their series of five cases of traumatic expulsion of posterior chamber intraocular lenses described the triad of expulsion of the lens, iris damage and intraocular haemorrhage all of which were seen in our patient. They also found in experimentation on donor human eyes that 0.68 joules of energy, which is well below that encountered in common environmental situations, could induce this injury. ${ }^{4}$

In a clinicopathological study of ocular trauma in eyes with intraocular lenses Assia et al found, as in this case, that falling was the most common trauma to precipitate globe rupture and artificial lens extrusion. They also noted that rupture usually occurred at the preexisting surgical wound site. ${ }^{2}$ Their study suggested that the eye with a posterior chamber lens was more resistant to trauma than eyes with the other types of artificial lenses. They felt that modern surgical techniques such as 
small incisions and scleral-tunnel wound construction could be expected to render the modern pseudophakic eye more durable. ${ }^{2}$

Wolter in 1963 paraphrased Courville's coup-contrecoup mechanism of craniocerebral injuries to explain the mechanism of ocular injury in blunt trauma. $\mathrm{He}$ ascribed lens displacement to a contre-coup injury. ${ }^{5-7}$ The lens is affected as it acts as an interface between the anterior chamber and posterior chamber, thus a wave of force as imparted by a blunt trauma can disrupt tissues of different densities. $^{5}$

The dislocated intraocular lens instead of being extruded through the surgical wound may dislocate into the vitreous cavity, anterior chamber, or the suprachoroidal space. A case report of a posterior chamber intraocular lens being dislocated into the anterior chamber while the surgical wound remained intact is described in the literature. ${ }^{8}$

Dislocation of an intraocular lens implant may be complicated by intraocular haemorrhage and endophthalmitis as well as other sequelae of blunt ocular trauma including corneal abrasion and oedema, subconjunctival haemorrhage raised intraocular pressure, iridodialyses, ciliary body necrosis, retinal necrosis, traumatic maculopathy, macular hole formation and retinal detachment. ${ }^{23}$

Investigations that may be of help in this condition include computed tomography of the orbit and B scan ultrasonography. ${ }^{359}$

Lens dislocation after trauma in the pseudophakic eye has been well described. Current ophthalmic practice for cataract surgery involves phaco-emulsification techniques in the large majority of patients. This procedure involves small scleral or corneal sections with the insertion of a foldable lens and subsequently lens extrusion after blunt trauma will be less commonly seen. However, where it does happen, as in our patient, modern anterior segment reconstruction techniques provide a favourable prognosis.

\section{Conclusion}

Our case represents the extrusion of the lens through a dehisced corneal section after blunt injury. The importance of protecting the eye after injury with an eye shield to prevent further intraocular extrusion due to direct pressure cannot be over emphasised. Many patients in the past have and a minority still undergo extracapsular extraction with wide corneal sectioning, emphasising the importance of recognising this increasingly rare complication that may be unfamiliar to many emergency physicians.

Contributors

Dr P Gilligan initially treated the patient and drafted the case report. Dr M J Gallagher was involved in the management of the patient and provided the expert ophthalmology details and cowrote the paper. Dr M Dudley and Dr P Cryer suggested alterations and additions to the case report.

Funding: none.

Conflicts of interest: none.

1 Volkov VV, Dal' GA, Tulina VM, Kulikov VS, et al. Contuion ruptures of the ocular capsule along post-operative corneo-limbic scars. Vestn Oftalmol 1998;114:17-20.

2 Assia EI, Blotnick Ca, Powers TP, et al. Clinicopathologic study of ocular trauma in eyes with intra-ocular lenses. Am 7 Ophthalmol 1994;117:30-6.

3 Canavan YM, Archer DB. Anterior segment consequences of blunt ocular injury. Br f Ophthalmol 1982;66:549-55.

4 Margal LE, Shakin E, Bolling JP, et al. Traumatic extrusion of posterior chamber lenses: clinical and experimental correlations. 7 Cataract Refract Surg 1986;12:670-3.

5 Giovinazzo VJ. The ocular sequelae of blunt trauma. $A d v$ Ophthalmic Plast Reconstr Surg 1987;6:107-14.

6 Wolter JR. Coup-contrecoup mechanism of craniocerebral injuries. Arch Surg 1942;45:19-43.

7 injuries. Arch Surg 1942;45:19-43. Courville CB. Coup-contrecoup mecha
injury. Am f Ophthalmol 1963;56:785-96.

8 Superstein R, Gans M. Anterior dislocation of a posterior chamber intra-ocular lens after blunt trauma. $\mathcal{F}$ Cataract Refract Surg 1999;25:1418-19.

9 Sathish S, Chakrabarti A, Prajna V. Traumatic subconjunctival dislocation of the crystalline lens and its surgical management. Ophthalmic Surg Lasers 1994;30:684-6. 